\title{
New simple optical sensor: from nanometer resolution to centimeter displacement range
}

\author{
A. Missoffe, L. Chassagne*, S. Topçu, P. Ruaux, B. Cagneau, Y. Alayli \\ Laboratoire d'Ingénierie des Systèmes de Versailles, Université de Versailles Saint-Quentin, 45 avenue des \\ Etats-Unis, 78035 Versailles, France \\ Corresponding author: luc.chassagne@uvsq.fr +33139254984 \\ Exact reference : \\ A. Missoffe, L. Chassagne, S. Topsu, P. Ruaux, B. Cagneau, Y. Alayli, New simple optical sensor: from \\ nanometer resolution to centimeter displacement range, Sensors and Actuators A, 176, 46-52 (2012).
}

\begin{abstract}
A very simple displacement sensor is presented with nanometric resolution over centimetric travel range. The compact system is composed of a laser-diode module and a photodiode array leading to a non-contact sensor. With a corner cube configuration, it is not sensitive to most of main mechanical defects of the mobile platform. The use of an optical fiber and a normalization process inspired from classical fourquadrant detectors allows high repeatability and minimal drifts. This paper exposes the setup, simulations and experimental results. Several displacements over millimeters range with a resolution of $1.2 \mathrm{~nm}$ sustain the simulations.
\end{abstract}

Keywords: high resolution sensor, nanodisplacement, distance measurement, low-noise electronics, photodetector

\section{Introduction}

With typical feature size of tens of nanometers over $300 \mathrm{~mm}$, the semiconductor industry has reached an unprecedented resolution versus working area. $32 \mathrm{~nm}$ nodes on $450 \mathrm{~mm}$ wafer are foreseen for 2012. Such resolution over centimeter range has been made possible using interferometric translation stages integrated in vacuum electron beam microscope, in other words by combining metrology with classical mechanical motion. Most of fields in modern sciences, and especially nanotechnology, need sensors that overcome the limitation of high resolution over long range of measurement.

The interferometry is obviously a good solution that combines sub-nanometer resolution over centimeter range. Interferometers have also the convenience to be accurate and traceable standards for displacement measurements. Nevertheless their sensitivity to the medium index and their price make them prohibited in many applications. In parallel of multiple works over interferometry, several researches have been launched to develop simple sensors or measurement systems gathering simplicity, high resolution over long range and low price potentialities. Most of the emerging systems have difficulties to combine nanometer resolution over centimeter range because these two characteristics are mainly opposite. However a lot of challenges in nanotechnology for future years are still dependant of easy-to-use instruments measuring displacement at nanometric level. Sensors are also fundamental in closed loop control systems. The capability to develop integrated and compact apparatus is still a key point for all the systems of control, displacement and manipulation in the atomic world [1-3].

Encoders are the main rivals of interferometry. Invar or zerodur gratings can be produced over centimetric range with periods of hundreds of nanometers. High performance encoders ensure nanometric resolution 
over centimetric ranges with high repeatability and very low periodic non-linearities [4], but to the detriment of the price. Fabry-Perot interferometers present high ratio range over resolution. Some of them reach sub-nanometric uncertainties over centimeters but to the detriment of the complexity [5]. Inductive sensors have been also developed but nanometric resolution remains difficult to reach because of magnetic disturbances. Capacitive sensors are more robust systems but it is quite difficult to obtain a good resolution over range ratio. Construction and mechanical care are very critical; nevertheless nanometric performances over millimetric range are possible [6]. The main drawback is the low bandwidth because capacitive phenomenon is quite a slow process. Coupling fiber systems have also been hardly developed since several years. Very low price and nanometric performances can be reached with an emitting fiber coupled with collecting fibers. High resolution [7] or high bandwidth [8-9] are possible. The intrinsic range of these sensors is limited to few hundreds of micrometers but combined with mechanical design it could reach the millimeter scale [10]. But long term drifts limit the performances of fiber systems because it is difficult to dispose of a stable reference. Triangulation optical systems combining light source, optical focusing mount and CCD elements (Charge Coupled Device) have been also developed. Based on the deviation of a retrodiffused signal and its detection on a CCD element, this kind of apparatus perform measurements over millimetric ranges with bandwidth of tens of kilohertz but the CCD elements limits the signal to noise ratio and resolution can not be at the nanometer level.

This paper presents an optical sensor for centimeter range measurements with nanometric resolution. The principle is described in a first part. Simulations of the signal to noise ratio are developed to explain the choice of optical and electronic parameters. The experimental setup is described and experimental results demonstrate the performances of the system, according to modelling results. The output signals of the sensor, in two configurations, illustrate the normalization process to ensure low drifts. The resolution has been estimated by measuring the noise level depending on the integration time. Repeatability and overall performances and advantages of the system are finally discussed. We plan to use this sensor with a nanodisplacement platform for nano-manipulation and for a sample-holder in microscopy or lithography process [11-12].

\section{Experimental setup}

\subsection{Principle of the sensor}

The sketch of the sensor is illustrated on Fig. 1a. The laser source is composed of a laser-diode module and a lens allowing the tuning of the focal length and the size of the spot at a distance of a few centimeters. An attenuating plate is also included to adjust the intensity of the spot, and to avoid reflection and feedback disturbances on the laser-diode. The sensitive part is a photodiode array (S4111-16R from Hamamatsu) composed of 16 adjacent photodiodes. The geometry of the photodiode elements is represented on Fig. $1 \mathrm{~b}$. Each photodiode has a height of $1.45 \mathrm{~mm}$ and a width of $900 \mu \mathrm{m}$, with a gap of $100 \mu \mathrm{m}$ between two elements. The size of the spot is tuned to be smaller than two elements in height and width (ellipsoidal shape for the example on the figure). The global principle of the sensor is like a four-quadrant photometry system, ie the detection of the displacement of a spot over the photodiodes. The 16 elements (the component exists also with 35 or 46 elements array) ensure the centimeter range, and the nanometric resolution is obtained thanks to the sensitivity of the photodiodes and the optimization of the signal to noise ratio. The signal to noise ratio calculus in the next paragraph is useful to choose the intensity of the spot and the parameters of the amplifiers.

The sensor can be adapted in two configurations. The first one, called direct configuration, where the emitting source is fixed, and the photodiode array is moving. In that case, the spot of the source illuminates directly the array. The second configuration is called corner cube configuration and is represented on Fig. 1a. The source and the receiver are fixed, and the moving part is composed of a corner cube. Both 
configurations have advantages discussed in the following. In the corner cube configuration, the sensitivity is multiplied by a factor of 2 thanks to the optical properties of the cube.

Each photodiode is connected to a transimpedance amplifier to convert the current to a voltage. The outputs of the amplifiers are measured with a high precision scanning voltmeter (Keithley 2700, 20 bits acquisition converters) controlled by a computer unit via a GPIB interface. For faster acquisition rates, the scanning voltmeter can be replaced by an NI data acquisition card (NI-PCI 4472, 24 bits, maximum sample frequency of $45 \mathrm{kHz}$ ). All the system is installed on a breadboard with vibration isolators and in a dark box to minimize thermal and lightning variations. Temperature is monitored each time measurements are recorded to control the stability of the elements.

To test the displacement performances, the mobile part is moved by a double stage actuator. The first stage is a piezo-electric actuator. It controls fine displacements of nanometer or micrometer range. This piezoelectric actuator is mounted on an air bearing stage moved by linear motors (ABL2000 from Aerotech). Its range of displacement is $10 \mathrm{~cm}$, with a repeatability of $0.2 \mu \mathrm{m}$ over the full range. The motion axis is referred as $x$ in the Fig. 1a.

\subsection{Simulation of the potential signal to noise ratio and resolution}

The resolution is limited by the signal to noise ratio of the measurement system, mainly by the signal to noise ratio of the signal at the photodiode array. The analysis of the noise sources and simulations of the signal to noise ratio are used to set up the parameters of the spot on the photodiode array. Each photodiode is connected to a trans-impedance electronics composed of a resistance $R$ and an operational amplifier (AOP). The classical noise sources are the shot noise of the photodiode current, the shot noise of the dark current, the thermal noise of the resistance, the Noise Equivalent Power (NEP) of the photodiode, the current noise of the AOP and the voltage noise of the AOP. All these noises are quadratically added according to noise models. The photodiode is not polarized, making the shot noise of the dark current mainly negligible. The model of the total noise has then several parameters: $R$ the resistance, $D$ the power density of the spot, $B$ the bandwidth considered and the size of the spot. Two types of spot are considered as a first approximation: round shape with a diameter $d$, and rectangular with a height $h$. Gaussian profile is considered in the second part of the paper for more complete modelling.

The signal to noise ratio depends on the position of the spot. Let us consider a spot moving along a photodiode element; $x$ is the position of the border of the spot. For a position from $1 \mathrm{~nm}$ to $900 \mu \mathrm{m}$, the full width of a photodiode element, the simulation calculates the signal depending on the position $x$ that induces a surface related to $x$, and the noise also related to $x$ by the shot noise. For each position $x$, we calculated the ratio expressed by Eq. 1

$$
S N R=\frac{S_{x}-S_{x+1}}{N},
$$

where $S_{x}$ is the signal at the position $x, S_{x+1}$ is the signal at the position $x+1 \mathrm{~nm}$, and $N$ is the noise. This method to normalize the signal to noise ratio verifies if a displacement of $1 \mathrm{~nm}$ is measurable. If $S N R>1$, a $1 \mathrm{~nm}$ displacement can be theoretically detected.

The Fig. 2 presents the results for two types of spot: round with a diameter $d=1 \mathrm{~mm}$, and rectangular with a height $h=1 \mathrm{~mm}$. It has been calculated for a density of the laser spot $D=0.05 \mathrm{~mW} / \mathrm{mm}^{2}$ and a bandwidth $B=50 \mathrm{~Hz}$. The resistance is $R=1 \mathrm{M} \Omega$ and the AOP is a precision amplifier OP27. The curves are inversely proportional to the square root of $B$. For the left part of the curve, one can see obviously that the rectangular shape is more appropriate than the round one. Indeed for the rectangular shape, the useful surface is immediately proportional to the height, and for the round shape, the useful part of the spot is only a tiny arc. For the rectangular spot and with $R=1 \mathrm{M} \Omega$, it is mainly the electronic noise that determines the signal to noise ratio on the left of the curve; when the spot is growing on the right of the curve, the 
electronic noise becomes at the same order of value than the shot noise of the source. The intensity of the laser spot can be adjusted; a power density of $D=0.05 \mathrm{~mW} / \mathrm{mm}^{2}$ has been chosen. Several sets of power density have been tested for measurements, showing that these values are a good compromise between the limitation due to electronics and laser source.

\section{Results and discussions}

Two sets of measurements are made. For the first one, a tilted attenuator is inserted after the laser diode to adjust the output power. For the second one, the beam is injected into a monomode fiber. The fiber selects one mode and the output intensity profile of the beam is therefore gaussian. For both configurations, simulations are made to predict the signals of the photodiodes. The results are then compared to measurements.

\subsection{Large displacement: without an optical fiber}

The beam profile of the laser diode is measured and the collected data are used to predict the signals on the photodiode array. Fig. 3 shows the measured beam profile along the horizontal direction $x$, using a beamscope (DataRay Inc., Beamscope-P7, $50 \mathrm{~nm}$ spatial resolution). The profile along the vertical $z$ direction is gaussian. The photodiode signals are simulated for a $1100 \mu \mathrm{m}$ wide spot with this intensity profile and to compare the result with measurements, a millimeter scale displacement is realized with the motor stage. The displacement is controlled by $5 \mu \mathrm{m}$ steps. At each step, the output of a photodiode is recorded by the electronics board (for the direct configuration, without a corner cube). Measurement is compared with the simulation on Fig.4. The signals approximately fit; both signals seem linear on a certain range and show non-linearities near the maxima and the minima. The difference is due to the fact that the beam profile used for the simulation is not exactly the same as the one of the measurements. Indeed, the laser diode is a cheap module and the shape of the intensity of the beam in Fig. 3 is submitted to drifts and real-time variations. Especially, the peaks on the left and right sides vary with environmental conditions. The simulation demonstrates that non-linearities near the maxima and the minima are due to the specific intensity profile with these peaks on the sides.

In a second step of the experiment, we did a displacement of more than $5 \mathrm{~mm}$. The curves for seven photodiode signals are measured and plotted on the Fig. 5. The direct configuration leads to Fig. 5a curves and the corner cube configuration leads to Fig. 5b curves. In the (a) case, the distance between two peaks is $1 \mathrm{~mm}$ and in the (b) case it is $500 \mu \mathrm{m}$ because of the factor 2 on the sensitivity illustrated on Fig. 1a. One can clearly see that when the spot is growing on a photodiode, an increasing slope is appearing. At the same time, it decreases on the adjacent photodiode on which it induces a negative slope.

From this set of curves, a normalization process is developed. For each couple of increasing and decreasing slopes, the Eq. (2) is applied to calculate the motion $\Delta x$ :

$$
\Delta x=k \cdot \frac{S_{n+}-S_{n-}}{S_{n+}+S_{n-}},
$$

where $S_{n+}$ is the signal of the increasing slope, $S_{n^{-}}$the signal of the decreasing slope and $k$ a function depending on the width and the intensity profile of the spot. The difference is taking advantage of the differential effect, and the division by the sum is a normalization classically used in photometry to eliminate variation of the intensity of the source. For a precise spot size of $1100 \mu \mathrm{m}$, it leads to a relative displacements of $1 \mathrm{~mm}$ normalized in the $[-1 ;+1]$ space and $k=2$. If one wants accuracy performances, $k$ has to be accurately calibrated. In the following of the paper, it has been done with the encoders of the mechanical stage for each plot. More accurate calibration could be done with an interferometer. But the 
signal is non-linear. One has to understand that, for the given spot size, even if the intensity profile is purely uniform, this signal could not be perfectly linear. There would be non-linear areas at both ends (near -1 and +1 ). The normalized signal is shown on Fig. 6 for the measurements as well as for the simulations with the intensity profile of Fig. 3 and a purely theoretical uniform intensity profile. The three curves match quite well but non-linearities and uncertainties remain. To illustrate that the shape of the signals is highly dependent on the size of the spot, the normalized photodiode signals are simulated for different spot sizes assuming that the intensity profile is the one on Fig. 3. The results are shown on Fig. 7. For a small spot size (width $<1 \mathrm{~mm}$ ), the normalized signal is quite linear but one can notice the presence of 'dead zones', for which the normalized signal does not vary with the displacement. This problem can be solved by using a larger spot (width $>1100 \mu \mathrm{m}$ ). One can then jump from a curve to another as one of the signals is valid to measure the displacement. But unfortunately, the larger is the spot, the more non-linear are the normalized amplitudes. So the instrument needs to be calibrated anyway.

\subsection{Large displacement: with an optical fiber}

To avoid the sensitivity of the sensor response over the intensity profile of the beam, the laser diode is injected in a monomode optical fiber which ensures a steady gaussian profile intensity at the output of the fiber. A lens at the output collimates the beam before the tilted attenuator. Samely to the condition of Fig. $5 \mathrm{a}$, a long-range displacement is done and a set of measurements over seven photodiodes elements is recorded. The signals are plotted on Fig. 8. The signals actually happen to be gaussian. The Fig. 9 shows the fitting with a gaussian profile where a correlation coefficient of 0.99986 is obtained which is very close to 1 .

Fig. 10 shows the superposition of the photodiode 5 signal and the signal predicted by simulations for a spot with an $1100 \mu \mathrm{m}$ standard deviation gaussian beam profile. The simulations confirm that the signal is gaussian. Actually the larger the spot is, the more gaussian are the signals from the photodiodes as illustrated by the simulation on Fig. 11. The width of the spot is considered to be three times the standard deviation of the gaussian beam profile; this corresponds to $95 \%$ of the emitted power.

These gaussian profiles are of course non-linear, but the insertion of the fiber allows high stability of the intensity profile. Furthermore, the form of the response signal is well-known with mathematical approximation of the gaussian fit, unlike the previous response with the profile of the Fig. 3. The Fig. 12 illustrates the normalized outputs of the sensor (using Eq. 2). Theoretically, the normalized signal varies from -1 to +1 as the first photodiode is illuminated and not the second one and vice versa. In practice, the signals do not reach these limits and continue to vary. Nevertheless one can see that we can always use one of the normalized signals to measure the displacement and 'dead zones' have disappeared (following the arrows on the graph). This is one main advantage to this configuration. Despite nonlinearities, the value of the normalized amplitude can be expressed as an analytical function of the displacement as the raw signals happen to be Gaussian; consequently there is no need for calibration.

\subsection{Resolution}

The resolution is mainly limited by the noise of the system, either the electronic noise or the shot noise of the spot. Measurements are started to verify that it can intrinsically reach the nanometer level. The mechanical stage is moved to adjust the spot to be at mid-slope on channels 3 and 4 (respectively decreasing and increasing slopes). The system is then locked on a static position. The outputs of the sensors are recorded and the normalization process performed. Each point of measurement is integrated over an integration time $\tau$ by the Keithley system or the data acquisition card. A large set of data is recorded so that the position is measured for several seconds or minutes. If the distribution of the set of data is gaussian 
shape, the standard deviation is the resolution of the measurement, illustrated on Fig. 13 for several integration times.

For the unfibered option, the Keithley is used with an integration time of $10 \mathrm{~ms}$; for the fibered option, the acquisition card is used with a sample frequency of $45 \mathrm{kHz}$, which leads to a larger set of data and negligible error bars on the graph. Whatever the acquisition system, one can see that with a $100 \mathrm{~ms}$ integration time, a resolution of $1.2 \mathrm{~nm}$ is reached, and the flicker noise is around $1.1 \mathrm{~nm}$. Note that these measurements have been done in the direct configuration. In the corner cube configuration, the resolution would be twice better, ie $0.6 \mathrm{~nm}$. These results confirm the theoretical calculus presented at the beginning of the paper. Other sets of measurements have been done while adjusting the power density to identify the main limiting parameter on the resolution. Although the signal could be increased by choosing high power density, the shot noise is not the limiting factor, and parameters are a compromise between gain, bandwidth, saturation of the amplifier, thermal noise and quantification noise. The noise of the electronics is very dependent on the realization of the electronics board, and even if sub-nanometric resolution has been obtained in favorable cases, the nominal limit seems to be at the nanometric level. Notice that subnanometric performances could be obtained with lever effects like in cantilever detection in near-field microscopy, but to the detriment to the accuracy because of the uncertainty on the deflection angle.

\subsection{Repeatability}

The repeatability of the sensor is the major characteristic for the aimed applications. The mechanical stage is controlled to perform back-and-forth steps of $400 \mu \mathrm{m}$ or $100 \mu \mathrm{m}$. The sensor records the displacement, simultaneously with an interferometer (from Sios company - 3 beams). The Fig. 14 illustrates a part of the $400 \mu \mathrm{m}$ steps run. The height of the steps is calculated for all the set of data and its standard deviation that is considered to be the repeatability of the sensor. The result is $\sigma=6.3 \mathrm{~nm} \pm 2.2 \mathrm{~nm}$. It is fully compatible with the performance of the mechanical stage, which is $0.2 \mu \mathrm{m}$ over the full displacement range of $10 \mathrm{~cm}$.

\subsection{Errors}

The sensor is not sensitive to the refractive index of the medium like all the interferometric sensors. In the corner cube configuration, it is also not sensitive to yaw and straightness defects because of the optical geometric properties of the corner cube. In this configuration, the optical path remains constant whatever the position of the cube. Therefore, the size of the spot on the photodiode array remains the same. Furthermore, if the beam direction and the photodiode array are not exactly perpendicular, a cosine error occurs, but it remains very small (proportional to $\alpha^{2} / 2$ where $\alpha$ is the misalignment angle). It is an important point because the corner cube has not to be high quality so it is not crucial if the output beam is not exactly parallel to the input beam. The main error remains the nonlinearities on the slopes but with the fiber option, the shape of the output is well known in theory.

\section{Conclusion}

A high sensitive displacement sensor has been developed with nanometric resolution and centimetric range. The principle is quite simple and the system can be easily mounted with cheap components. Nanometer resolution has been experimentally verified and repeatability also. A configuration with corner cube allows the system to be not sensitive to several mechanical defects. This work is a part of an integrated project in the nanosciences field where this kind of sensor is an interesting alternative for interferometric system. We plan to use this system in a nano-displacement platform for nano-manipulation and for a sample-holder in microscopy or lithography process that has already demonstrates high interests for nanometrology [11-12]. 


\section{Acknowledgments}

This research was supported by the French research ministry (ANR-Pnano program). Authors thank Frédéric Mourgues for mechanical support and Justine Mignot for all the experimentation works and results.

\section{References}

[1] A. Engel, Nature's building blocks, Nanotechnology 20 (2009) 430202.

[2] M. Miles, Probing the nanoworld, Nanotechnology 20 (2009) 430208.

[3] J. Brugger, Nanotechnology impact on sensors, Nanotechnology 20 (2009) 430206.

[4] A. Yacoot, N. Cross, Measurement of picometre non-linearity in an optical grating encoder using x-ray interferometry, Meas. Sci. Technol. 14 (2003) 148-152.

[5] M. Durand, J. Lawall, Y. Wang, High-accuracy Fabry-Perot displacement interferometry using fiber lasers, Meas. Sci. Technol. 22 (2011) 094025.

[6] M. Kim, W. Moon, E. Yoon, K-R. Lee, A new capacitive displacement sensor with high accuracy and long-range, Sens. Actuators A 130-131 (2006) 135-141.

[7] M. Yasin, S.W. Harun, K. Karyono, H. Ahmad, Fiber optic displacement sensor using a multimode bundle fiber, Microwave Opt.Technol. Letters 50 (2008) 661-663.

[8] R. Dib, Y. Alayli, P. Wagstaff, A broadband amplitude-modulated fiber optic vibrometer with nanometric accuracy Measurement 35 (2004) 211-219.

[9] A. Khiat, F. Lamarque, C. Prelle, P. Phouille, M. Leester-Schädel, S. Büttganbach, Two-dimension fiber optic sensor for high-resolution and long-range linear measurements, Sens. Actuators A 158 (2010) 43-50.

[10] L. Perret, L. Chassagne, S. Topcu, P. Ruaux, B. Cagneau, Y. Alayli, Fiber optics sensor for subnanometric displacement and wide bandwidth systems, Sens. Actuators A, 165 (2011) 189-193.

[11] L. Chassagne, S. Blaize, P. Ruaux, S. Topcu, P. Royer, Y. Alayli, G. Lérondel, Multi-scale Scanning probe Microscopy, Rev. Sci. Instrum., 81 (2010) 086101.

[12] G. Lérondel, A. Sinno, L. Chassagne, S. Blaize, P. Ruaux, A. Bruyant, S. Topçu, P. Royer, A. Alayli, Enlarged near-field optical imaging, J. Appl. Phys. 106 (2009) 044913. 


\section{Vitae:}

Alexia Missoffe received her Engineer degree from Supelec, France, in 2005, as well as a Msc Degree in Microsystems engineering from Heriot Watt University, Edinburgh, UK. She received her Ph.D. degree from Ecole Centrale Paris and Supelec, France, in 2010 on Microsystems modelling. In 2010, she joined the LISV for a postdoctoral position to work on precision displacement sensors for AFM instrumentation.

Luc Chassagne is graduated from Engineer degree of Supelec (France) in 1994 and received his Ph.D. in optoelectronics from the University of Paris XI, Orsay, France in 2000 for his work in the field of atomic frequency standard metrology. He is now Professor at the LISV and the topics of interest in its research are nanometrology, precision displacements, sensors and AFM instrumentation.

Suat Topçu received his PhD from Compiègne University of Technology (Compiègne, France) in 2001. Since 2002, he works as an assistant professor at the University of Versailles in LISV laboratory. His fields of research are interferometry, dimensional metrology, ellipsometry and recently laser cooling and trapping process. He is professor at LISV since 2010.

Pascal Ruaux received the Ph.D. degrees in Robotic from the University of Pierre \& Marie Curie-Paris 6, Paris, France in 1998 to LRP (Laboratoire de Robotique de Paris). He became Associate Professor in 2001 with the LISV. He worked on technical assistant to disability. Since 2006, the centers of interest are control micro- and nano-precision on millimeter displacement, AFM large image and nano-manipulation.

Barthélemy Cagneau received the Ph.D. degrees in mechanical engineering from the University of Pierre \& Marie Curie-Paris 6, Paris, France in 2008. After a postdoctoral position in nano-robotics at ISIR (Institut des Systèmes Intelligents et de Robotique, Paris), he became Associate Professor in 2009 with the LISV (Laboratoire d’Ingénierie des Systèmes de Versailles, Versailles). His research interests include force control, adaptive control, and robust bilateral couplings for micro- and nano-robotics.

Yasser Alayli received his $\mathrm{PhD}$ in applied physics from Pierre et Marie Curie University of Paris (Paris, France) in 1978. He is professor at Versailles Saint-Quentin University, France, and director of the LISV. His research interests include precision engineering domain with sub-nanometric accuracy and nanotechnologies. 


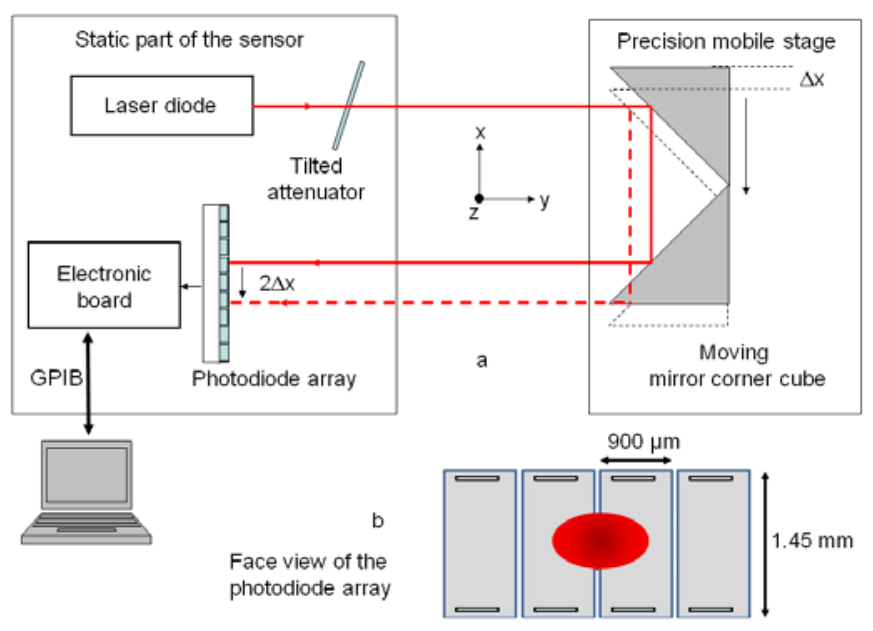

Figure 1 


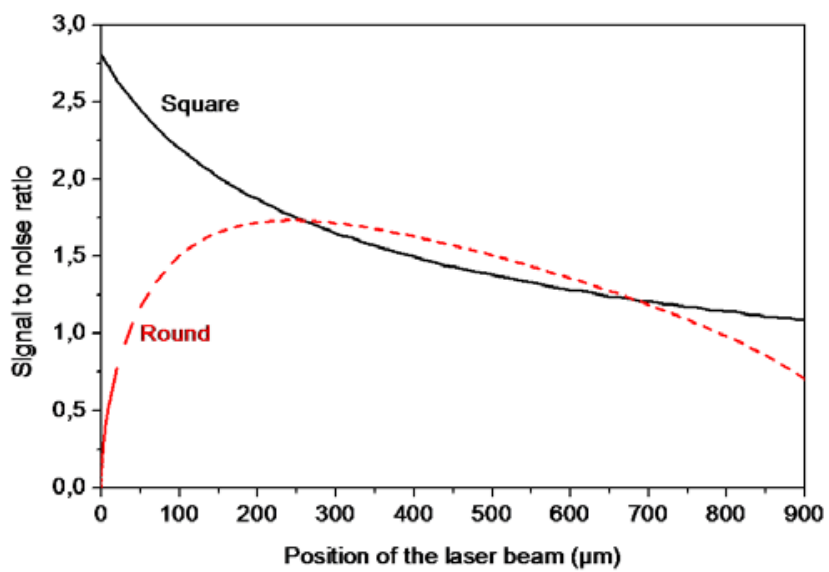

Figure 2 


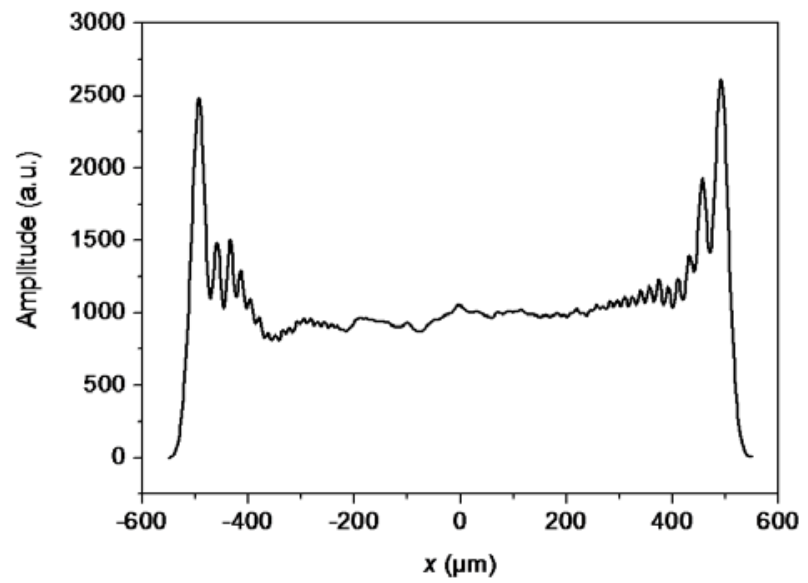

Figure 3 


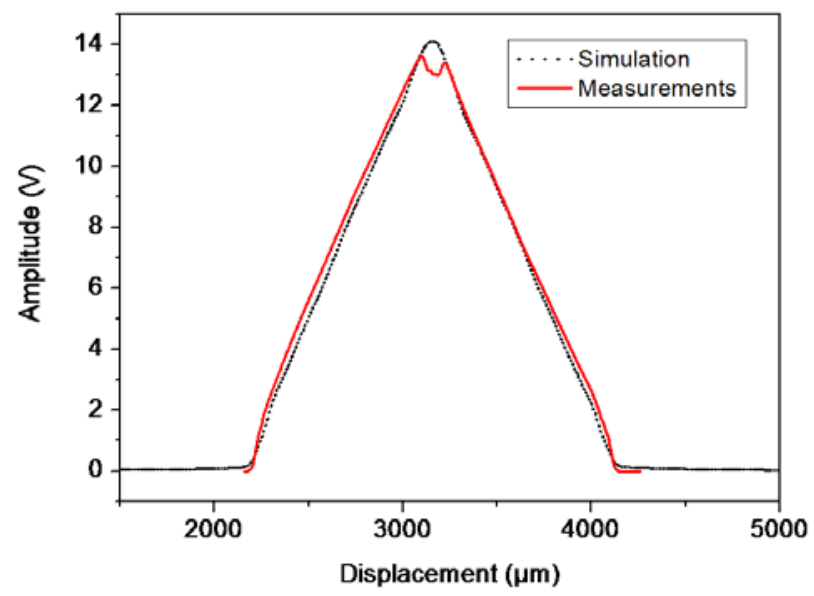

Figure 4 
a

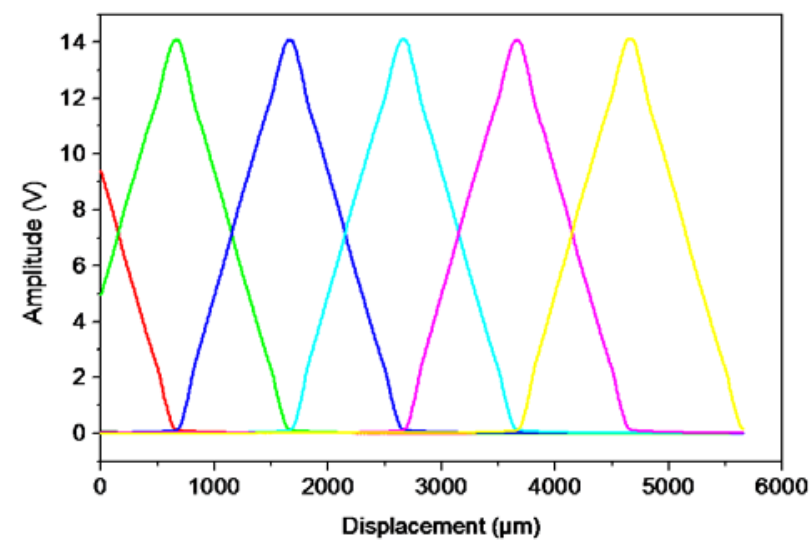

b

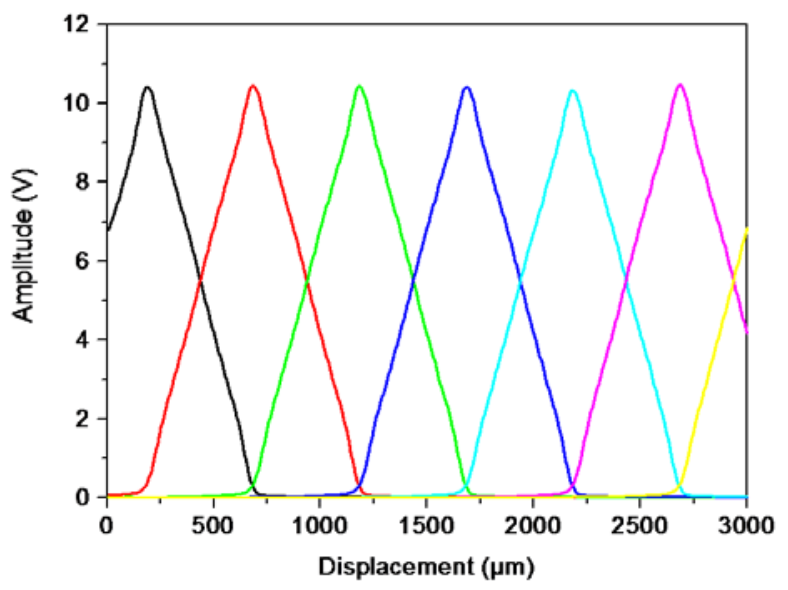

Figure 5 


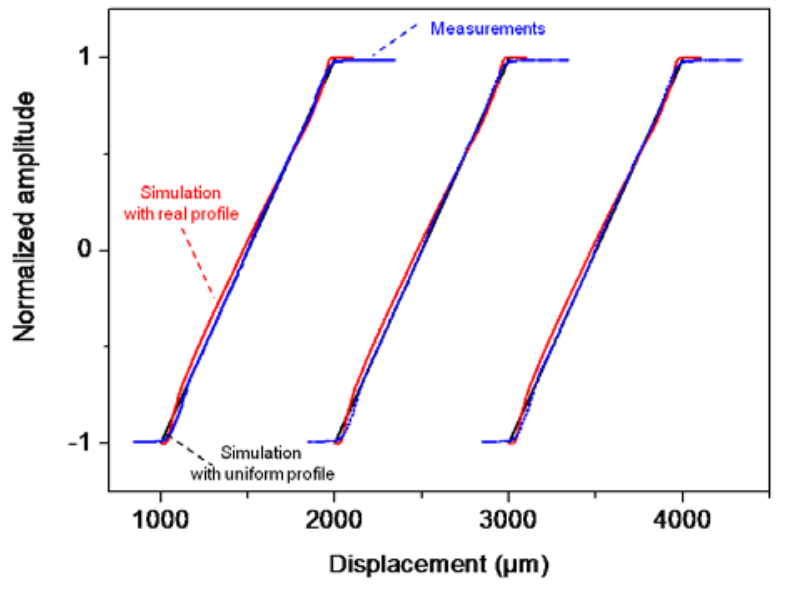

Figure 6 


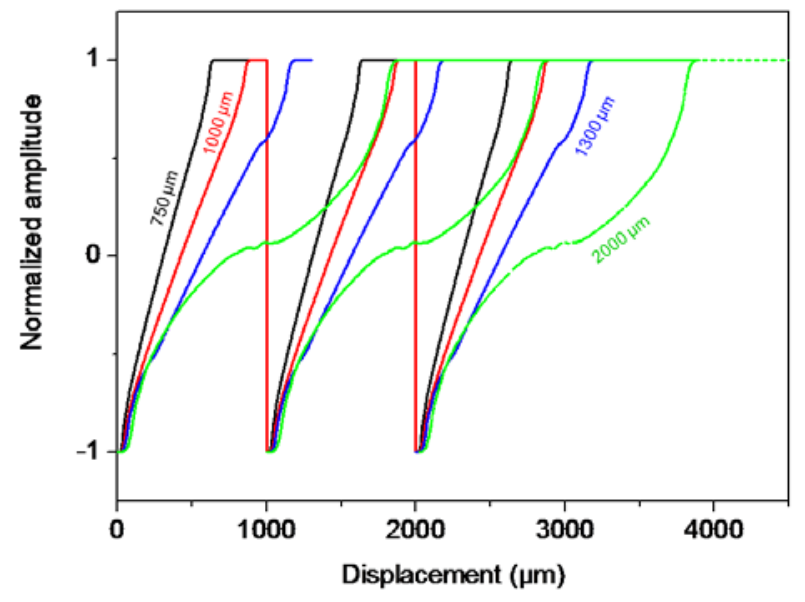

Figure 7 


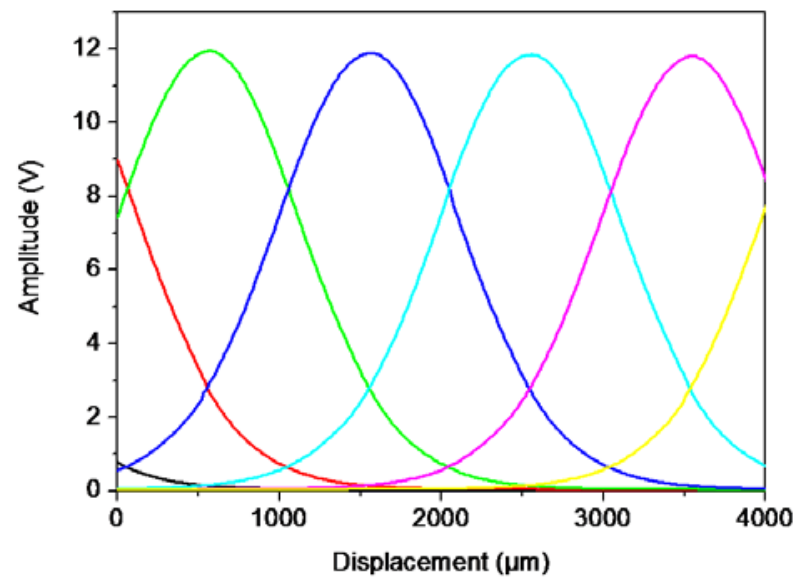

Figure 8 


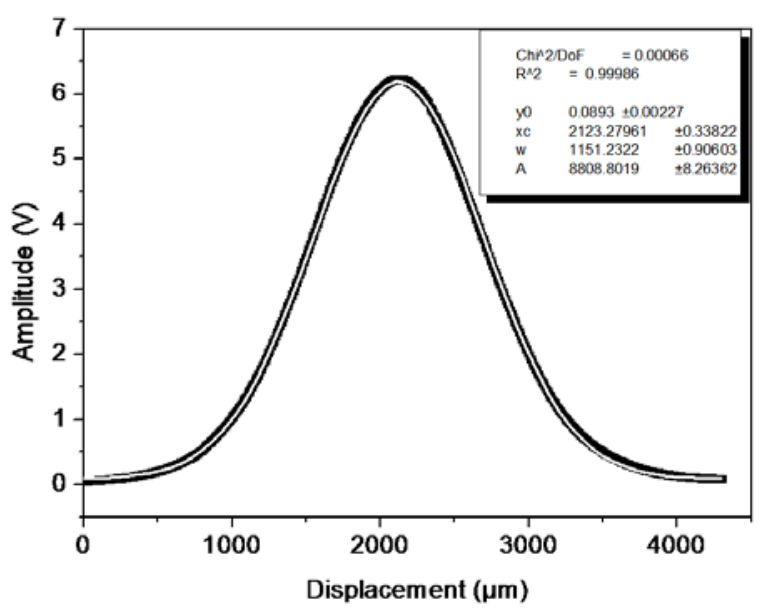

Figure 9 


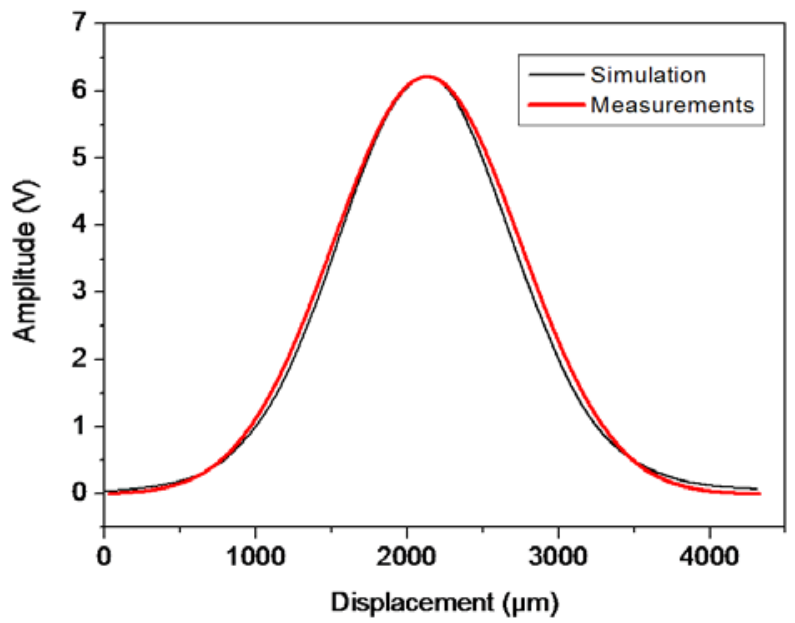

Figure 10 


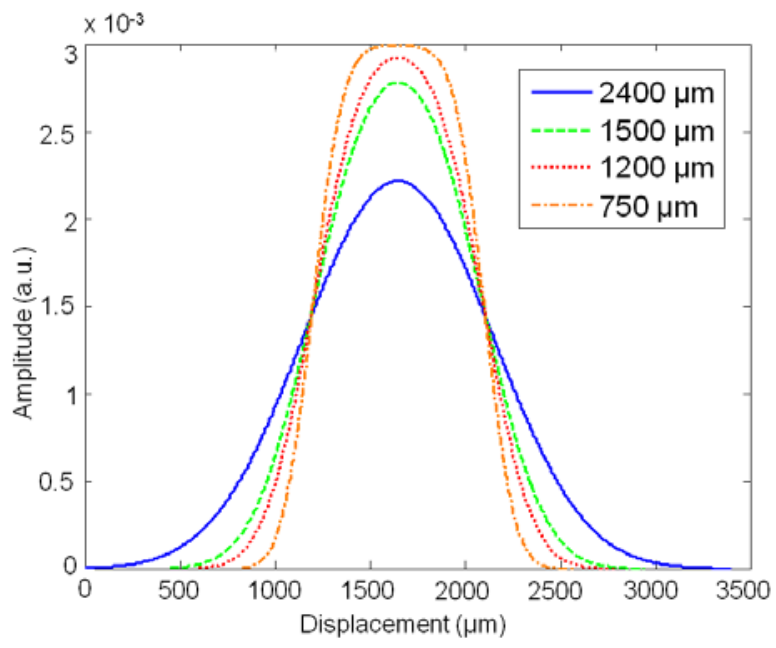

Figure 11 


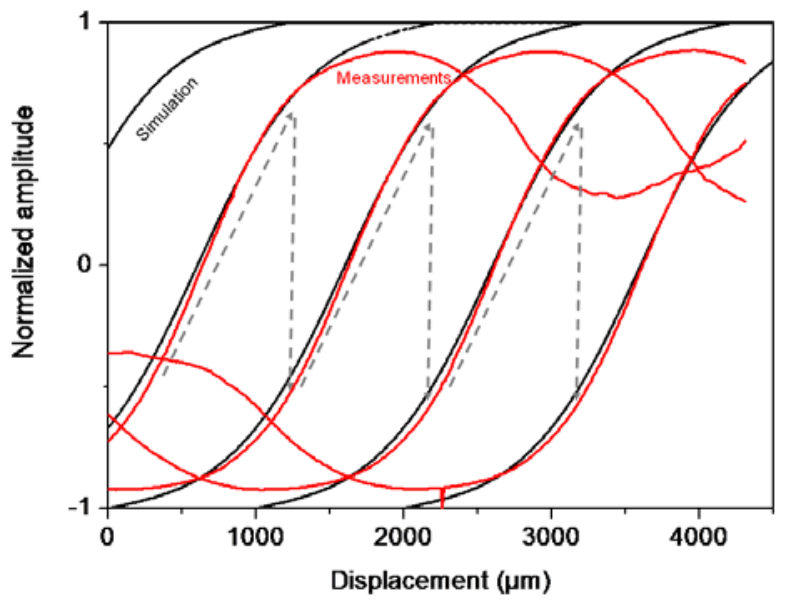

Figure 12 


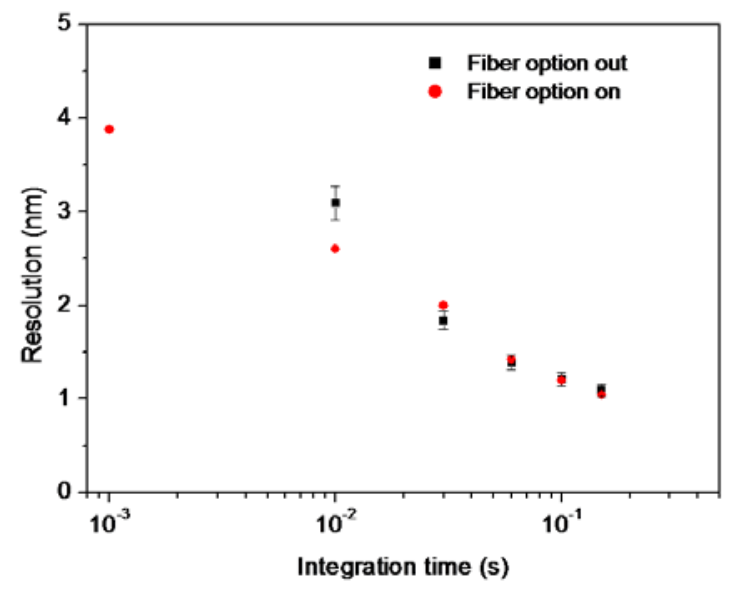

Figure 13 


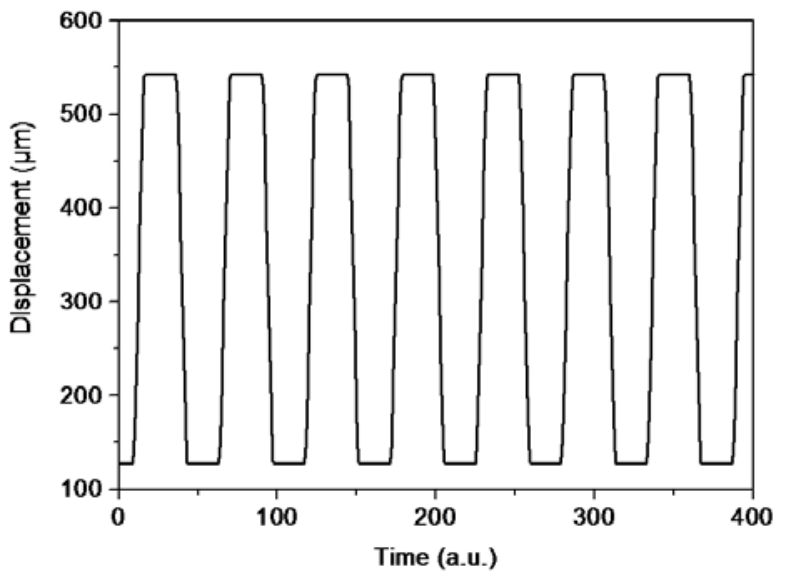

Figure 14 


\section{Figure captions}

Fig. 1. Schematic diagram of the sensor in the corner cube configuration. (a) The sensor is composed of a static part: laser diode source and tilted attenuator for the emitting elements, and photodiode array and electronics for conditioning signal board; the mobile part is made of a corner cube. (b) Face view of the photodiode array and a theoretical rectangular shape spot for clarity.

Fig. 2. Simulations of the signal to noise ratio for two different shapes of the spot. The signal to noise ratio is expressed like the difference signal corresponding to a $1 \mathrm{~nm}$ displacement divided by the noise of the signal. If it is higher than 1 , it induces that a $1 \mathrm{~nm}$ displacement is visible. This set of curves is calculated with a power density of the spot of $0.05 \mathrm{~mW} / \mathrm{mm}^{2}$ and are inversely proportional to the square root of the bandwidth (50 $\mathrm{Hz}$ in this example).

Fig. 3. Measured beam profile of the laser diode module along the horizontal direction $x$.

Fig. 4. Simulation of one photodiode signal versus displacement knowing the beam intensity profile for a $1100 \mu \mathrm{m}$ wide laser spot and comparison with measurements.

Fig. 5. Measurement of the displacement over millimetric range; (a) is for direct configuration and (b) for corner cube configuration. The factor of 2 on the sensitivity is clearly appearing by the expansion of the abscissa scale.

Fig. 6. Normalized amplitude for the measurements, simulations with the intensity profile of Fig. 3, and simulations with an uniform intensity profile (spot width $1100 \mu \mathrm{m}$ ).

Fig. 7. Simulation of normalized amplitude for different spot sizes, the profile intensity being the one of Fig. 3.

Fig. 8. Long-range displacement measurements with an optical fiber inserted after the laser diode module.

Fig. 9. Gaussian fit of the photodiode signal: black points are the measurements and white line is the gaussian fit with the parameters in the insert.

Fig. 10. Measured and simulated signals assuming a $1100 \mu \mathrm{m}$ standard deviation gaussian beam profile.

Fig. 11. Simulated signals for different spot sizes. The $x$ axis is not exactly the displacement as the curves are centred to enable the shape comparison. The larger the spot is, the more gaussian is the response.

Fig. 12. Normalized amplitude versus displacement for simulated and experimental results.

Fig. 13. Measurements of the resolution of the sensor depending on the integration time. These measurements have been done on the direct configuration without corner cube. It would be twice better with the cube configuration.

Fig. 14. Measurements of the repeatability of the sensor with $400 \mu \mathrm{m}$ steps. 